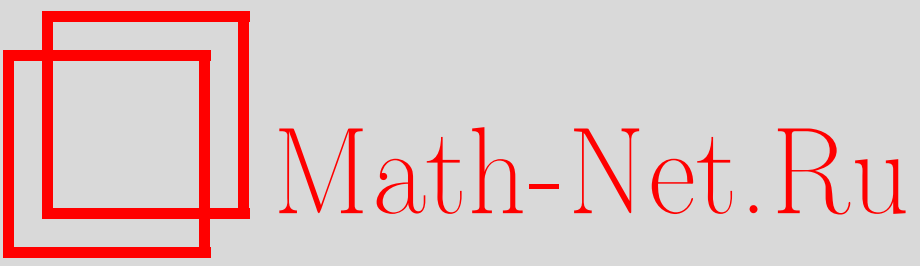

А. Я. Казаков, С. Ю. Славянов, Интегральные соотношения для специальных функций класса Гойна, ТМФ, 1996, том 107, номер 3, 388-396

DOI: https://doi.org/10.4213/tmf1164

Использование Общероссийского математического портала Math-Net.Ru подразумевает, что вы прочитали и согласны с пользовательским соглашением

http://www.mathnet.ru/rus/agreement

Параметры загрузки:

IP: 54.89 .56 .158

26 апреля 2023 г., 16:01:44 


\author{
ТЕОРЕТИЧЕСКАЯ \\ И МАТЕМАТИЧЕСКАЯ \\ ФИЗИКА \\ Том 107, № 3 \\ июнь, 1996
}

А.Я. Казаков, С.Ю. Славянов

\title{
ИНТЕГРАЛЬНЫЕ СООТНОШЕНИЯ ДЛЯ СПЕЦИАЛЬНЫХ ФУНКЦИЙ КЛАССА ГОЙНА
}

Получены новые интегральные соотношения для собственных функций, порожденных уравнениями класса Гойна. Эти соотношения выражают двойственность между собственными функциями, соответствующими уравнениям с разным поведением в особых точках и, возможно, заданными на разных интервалах. Рассматриваемые соотношения образуют две иерархии, в каждой из которых порождающие уравнения связаны друг с другом процессом конфиююенции особых точек.

\section{1. ВВЕДЕНИЕ}

Уравнение Гойна - обыкновенное линейное дифференциальное уравнение (ОДУ) второго порядка с четырьмя регулярными особенностями. Вместе с уравнениями, которые получаются из него с помошью предельных переходов или заданием специальных значений параметров, оно образует класс Гойна дифференциальных уравнений $[1,2]$. Специальным образом выбранные решения уравнений класса Гойна порождают класс Гойна специальных функций [2].

Цель данной работы - описание и исследование интегральных соотношений, связываюших между собой собственные функции, порождаемые уравнениями класса Гойна с разными наборами характеризуюших их параметров. Здесь мы не обсуждаем интегральные уравнения для собственных функций, отсылая к нашей предыдушей публикации [3].

Искомыми являются интегральные соотношения вида

$$
y(z)=\int_{C} K(\phi(z, t)) v(t) d t .
$$

Предполагается, что ядро $K(\phi)$ выражается в терминах элементарных функций, функция $\phi(z, t)$ - один из простых мономов

$$
\begin{aligned}
& \phi_{1}(z, t)=z t, \\
& \phi_{2}(z, t)=z-t,
\end{aligned}
$$

где $C$ - подходяший контур интегрирования на комплексной плоскости $t$. Функции $y(z)$, $v(t)$ в контексте данной статьи являются собственными функциями какой-либо граничной задачи для соответствующего уравнения класса Гойна. 
Полученные результаты можно использовать в следующих целях:

а) вычисление интегралов от специальных функций класса Гойна,

б) изучение свойств матриц монодромии,

в) вычисление значений специальных функций класса Гойна.

Специальные функции класса Гойна широко используется в физике (задача двух кулоновских центров, эффект Штарка на водороде, ангармонический осциллятор и др.), поэтому наши результаты могут быть полезны в многочисленных приложениях.

\section{2. ПОДГОТОВИТЕЛЬНЫЕ СВЕ ДЕНИЯ}

В качестве первого шага нам необходимо систематическое описание класса Гойна. Это описание подобно приведенному в $[4,5]$ и основано на уточненной классификации особых точек и явном исследовании процесса конфлюенции особых точек.

Каждой особой точке $z_{j}$ линейного ОДУ второго порядка может быть поставлено в соответствие характеризующее ее целое или полуцелое число $R\left(z_{j}\right)$, которое называется $s$-рангом особой точки (см. $[4,5])$. По определению $s$-ранг регулярной особой точки равен единице. Из определения $s$-ранга иррегулярной особенности $z=\infty$ следует, что в ее окрестности есть по крайней мере одно решение $y_{1}(z)$ с асимптотикой

$$
\left(\ln y_{1}(z)\right)^{\prime} \sim \alpha_{1} z^{R-1}(1+o(1)) .
$$

Формула (4) дает практический метод для вычисления $s$-ранга иррегулярной особенности. У рассматриваемых ниже уравнений $s$-ранги особых точек лежат в интервале

$$
3 / 2 \leq R \leq 4
$$

Набор $s$-рангов особых точек данного уравнения образует его $s$-мультисимвол $[4,5]$.

Процесс конфиюенции, в котором две особые точки уравнения сливаются, образуя особую точку с бо́льшим $s$-рангом, может быть описан как предельньй переход, при котором некоторые параметры уравнения стремятся к бесконечности [5].

Класс Гойна содержит “по существу” следующие дифференциальные уравнения:

$$
\begin{aligned}
L_{z}^{(0)} y(z)= & \left(z(z-1)(z-a) D^{2}+(\gamma(z-1)(z-a)+\delta z(z-a)+\right. \\
& +\varepsilon z(z-1)) D+(\alpha \beta z-q)) y(z)=0,
\end{aligned}
$$

причем $\gamma+\delta+\varepsilon=\alpha+\beta+1$,

$$
\begin{aligned}
& L_{z}^{(1)} y(z)=\left(z(z-1) D^{2}+(p z(z-1)+\gamma(z-1)+\delta z) D+(p \alpha z-q)\right) y(z)=0 \\
& L_{z}^{(2)} y(z)=\left(z(z-1) D^{2}+(\gamma(z-1)+\delta z) D-\left(\kappa^{2} z+q\right)\right) y(z)=0 \\
& L_{z}^{(3)} y(z)=\left(z^{2} D^{2}+\left(-z^{2}+\tau z+\nu\right) D+(-\alpha z-q)\right) y(z)=0 \\
& L_{z}^{(4)} y(z)=\left(z^{2} D^{2}+(\rho z+\omega) D-(z+q)\right) y(z)=0 \\
& L^{(5)} y(z)=\left(z D^{2}+\left(-z^{2}+\mu z+\gamma\right) D+(-\alpha z-q)\right) y(z)=0 \\
& L_{z}^{(6)} y(z)=\left(z D^{2}+\gamma D+\left(-z^{2}-\zeta z-q\right)\right) y(z)=0 \\
& L_{z}^{(7)} y(z)=\left(D^{2}+\left(-z^{2}+\sigma\right) D+(-\alpha z-q)\right) y(z)=0 \\
& L_{z}^{(8)} y(z)=\left(D^{2}+\left(-z^{3}+\tau z^{2}-q\right)\right) y(z)=0 .
\end{aligned}
$$


Уравнения (6)-(14) отличаются своими мультисимволами, которые соответственно равны $\{1,1,1,1\},\{1,1,2\},\{1,1,3 / 2\},\{2,2\},\{2,3 / 2\},\{1,3\},\{1,5 / 2\},\{4\},\{7 / 2\}$. В (6)-(14) мы пытались сохранить единое обозначение $q$ для так называемого акцессорного параметра, который в отличие от остальных параметров уравнения не связан с локальным поведением решений в окрестности какой-либо особой точки.

Исходное уравнение (6) - это известное уравнение Гойна (УГ) $[1,4]$. Уравнение (7) называется конфлюентным уравнением Гойна (КУГ) $[1,4]$ и может быть получено из (6) с помощью процесса конфлюенции

$$
\varepsilon=-p a+\alpha+1-\delta-\gamma, \quad \beta=-p a, \quad q=-a q, \quad a \rightarrow \infty .
$$

В физических приложениях часто используются частные случаи КУГ - уравнение для кулоновских сфероидальных функций, уравнение для сфероидальных функций, алгебраическая форма уравнения Матье [2]. Если мы положим, что

$$
\beta=a^{1 / 2} \kappa, \quad \alpha=a^{1 / 2} \kappa, \quad q \mapsto-a q, \quad a \rightarrow \infty,
$$

то получим уравнение (8) с полуцелым $s$-рангом для особенности на бесконечности, которое назовем редуцированным КУГ.

В последующем в процессе конфлюенции либо сливаются две регулярные особые точки, либо регулярная особенность сливается с иррегулярной. В результате первого процесса мы получаем дважды конфлюентное уравнение Гойна (ДКУГ) $(9)[1,4]$

$$
z \mapsto-\frac{z}{p}, \quad \gamma=\frac{\nu}{p}, \quad \delta=-\frac{\nu}{p}+\tau, \quad p \rightarrow 0
$$

Во втором случае после преобразования

$$
z \mapsto \frac{z}{\sqrt{p}}, \quad \delta=p-\mu \sqrt{p}, \quad q \mapsto-q \sqrt{p}, \quad p \rightarrow \infty
$$

мы получаем биконфлюентное уравнение Гойна (БКУГ) $(11)[1,4]$.

Взяв в качестве исходного редуцированное КУГ (8), мы получаем в результате преобразования

$$
z \mapsto \frac{z}{\kappa^{2}}, \quad \delta=\frac{\omega}{\kappa^{2}}+\rho, \quad \gamma=-\frac{\omega}{\kappa^{2}}, \quad \kappa \rightarrow 0,
$$

вместо (9) уравнение (10), которое мы назовем редуцированным ДКУГ с полуцелым $s$-рангом на бесконечности. Чтобы получить из (11) уравнение (12) - редуцированное БКУГ с полуцелым $s$-рангом, нам необходимо сделать преобразование зависимой переменной $y(z) \mapsto z^{1 / 2-\delta / 2} y(z)$ и затем вьполнить предельный переход

$$
z \mapsto z \varepsilon, \frac{1-\delta}{2}=\varepsilon^{-3 / 2}, \kappa^{2}+\varepsilon^{-3}=-\zeta \varepsilon^{-1}, q \varepsilon-\varepsilon^{-2}-\gamma \varepsilon^{-1 / 2} \mapsto q, \varepsilon \rightarrow \infty
$$

Последнюю возможность предоставляет процесс слияния всех особых точек в одну иррегулярную особую точку. После преобразования

$$
z \mapsto \frac{\mu}{2}+\left(\frac{\mu}{2}\right)^{1 / 3} z, \quad \gamma=3 \mu^{2}+\left(\frac{\mu}{2}\right)^{1 / 3} \sigma, \quad q \mapsto\left(\frac{\mu}{2}\right)^{1 / 3}\left(q-\frac{\alpha \mu}{2}\right), \quad \mu \rightarrow \infty
$$


мы получаем триконфлюентное уравнение Гойна (ТКУГ) $(13)[1,4]$. Чтобы вывести уравнение (14) - редуцированное ТКУГ - из описанных выше, требуется выполнить предварительно преобразование зависимой переменной

$$
y(z)=\exp \left(z^{2} / 2-\mu z-\gamma \ln (z)\right) w(z)
$$

После этого уравнение (14) можно получить с помошью подстановки

$$
\begin{aligned}
z \mapsto a^{1 / 4}+z a^{5 / 4}, & \gamma=O\left(a^{5 / 2}\right), \\
\mu=O\left(a^{5 / 4}\right), \quad \alpha=O\left(a^{5 / 2}\right), & q=O\left(a^{15 / 4}\right), \quad a \rightarrow \infty .
\end{aligned}
$$

Ввиду громоздкости соответствующих выражений мы указали в (23) только порядок величины коэффициентов, а не явные формулы для них.

Рассмотрим соотношение между дифференциальными и интегральными операторами на формальном уровне. Это означает, что мы полагаем все встречаюшиеся интегралы сходящимися и все внеинтегральные члены, возникаюшие при интегрировании по частям, равными нулю. Этого можно достичь выбором подходяших условий для параметров уравнений и выбором контура интегрирования.

Лемма 1. Пусть $L_{z}$ и $M_{t}$ - линейные дифференциальные операторы с полиномиальными коэффициентами. Предположим, что функиии $y(z)$ и v(t) удовлетворяют уравнениям

$$
\begin{aligned}
& L_{z} y(z)=0, \\
& M_{t} v(t)=0
\end{aligned}
$$

и связаны друг с другом соотношением (1). Тогда ядро $K(z, t)$ может быть выбрано как решение уравнения в частных производных

$$
\left(L_{z}-f(z) M_{t}^{*}\right) K(z, t)=0
$$

c подходящими функииями $g(t)$ и $f(z)$.

ДоКАЗАТЕЛЬСТво леммы следует из преобразования, включающего интегрирование по частям,

$$
\begin{aligned}
L_{z} y(z) & =\int_{C} v(t)\left(L_{z}-f(z) M_{t}^{*}\right) K(z, t) d t+\int_{C} f(z) K(z, t) M_{t} v(t) d t= \\
& =\int_{C} v(t)\left(L_{z}-f(z) M_{t}^{*}\right) K(z, t) d t=0 .
\end{aligned}
$$




\section{3. ИНТЕГРАЛЬНЫЕ СООТНОШЕНИЯ ДЛЯ ФУНКЦИЙ КЛАССА ГОЙНА}

Рассмотрим соотношения типа

$$
y(z)=K^{(0)} v=\int_{C}(z-t)^{\mu} v(t) d t,
$$

где $y(z)$ и $v(t)$ являются решениями уравнения Гойна с разными наборами параметров: $\{a, \alpha, \beta, \gamma, \beta, \varepsilon, q\}$ и $\left\{a, \alpha^{\prime}, \beta^{\prime}, \gamma^{\prime}, \beta^{\prime}, \varepsilon^{\prime}, q^{\prime}\right\}$, соответственно.

ЛЕмма 2. Уравнение

$$
\left(L_{z}^{(0)}-M_{t}^{(0) *}\right)(z-t)^{-\mu}=0
$$

справедливо, если вышеупомянутые наборы параметров связаны друг с другом следующими сотношениями:

$$
\begin{gathered}
(\mu-\alpha)(\mu-\beta)=0, \quad \gamma^{\prime}=\gamma+1-\mu, \\
\delta^{\prime}=\delta+1-\mu, \quad \varepsilon^{\prime}=\varepsilon+1-\mu, \\
\alpha^{\prime}=2-\mu, \quad \beta^{\prime}=-2 \mu+\alpha+\beta+1, \\
q^{\prime}=q+(-\mu+1)(\varepsilon+\delta a+\gamma(a+1))+\mu(\mu-1)(a+1) .
\end{gathered}
$$

ДокАЗАТЕЛЬСТво состоит в последовательном сокрашении коэффициентов, стоящих при различных степенях переменных $z, t$.

Имеются два корня квадратного уравнения (30) для параметра $\mu$, но при любом выборе мы получаем один и тот же оператор $M_{t}^{(0) *}$. Это следует из симметрии УГ относительно параметров $\alpha, \beta$. Поэтому, не теряя общности, параметры УГ для функции $v(t)$ в (28) можно выбрать следующим образом:

$$
\begin{gathered}
\gamma^{\prime}=\gamma+1-\alpha, \quad \delta^{\prime}=\delta+1-\alpha, \quad \varepsilon^{\prime}=\varepsilon+1-\alpha, \quad \alpha^{\prime}=2-\alpha \\
\beta^{\prime}=\beta+1-\alpha, \quad q^{\prime}=q+\alpha(\alpha-1)(a+1)-(\alpha-1) .
\end{gathered}
$$

При повторном применении преобразования (28) вновь возникающие коэффициенты совпадают с исходными. Следовательно, на формальном уровне справедливо соотношение

$$
\left(K^{(0)}\right)^{2}=I,
$$

где $I$ - тождественный оператор, так что (28) - преобразование инволюции. Это следует также из того, что двукратная суперпозиция преобразования (28) имеет ядро Коши.

С помощью формального соотношения (28) можно получить несколько конкретных результатов, подходяшим образом выбирая решения $y(z), v(t)$ и коэффишиенты для соответствующих уравнений. Здесь мы обсудим только связи между собственными функциями. 
ИНТЕГРАЛЬНЫЕ СООТНОШЕНИЯ ДЛЯ СПЕЦИАЛЬНЫХ ФУНКЦИЙ КЛАССА ГОЙНА 393

Tеорема 1. Предположим, что $a<0, \delta>\alpha, \gamma>1, \varepsilon>1, \quad \alpha<1$. Пусть $\left\{v_{n}(t)\right\}$ - набор собственных функций для задачи на собственные значения на интервале $[0,1]$ для УГ (6) с коэффициентами $\alpha^{\prime}, \beta^{\prime}, \gamma^{\prime}, \delta^{\prime}, \varepsilon^{\prime}, q^{\prime}$, определенными в (30) $\left(v_{n}(t)\right.$ ограничена в точках 0 и 1$)$. Тогда соотночение

$$
y_{n}(z)=K^{(0)} v_{n}=\int_{0}^{1}(z-t)^{-\alpha} v_{n}(t) d t
$$

порождает набор собственных функций $y_{n}(z)$ задачи на собственные значения на интервале $[a, 0]$ для уравнения (6) ( $y_{n}(z)$ ограничена в точках 0 и а).

ДокАЗАТЕльСтво. Интеграл в (33) сходится, так как $z \in] a, 0[$, и ограничен в $z=a$. Он также ограничен в $z=0$, так как $\alpha<1$. Все члены, возникаюшие при интегрировании по частям, пропадают (в силу поведения решений в конечных точках контура интегрирования). Следовательно, $y_{n}(z)$, определенная соотношением $(33),-$ собственная функция, соответствуюшая интервалу $[a, 0]$. ( Мы не обрашаем внимания на нормировку собственной функции.)

После подстановки $t=a / s$ соотношение (33) преобразуется в интегральное соотношение с ядром, зависящим от произведения $z s$. По некоторым причинам мы изменим интервал интегрирования.

Теорема 2. Предположим, что $a>1, \alpha>\gamma, \beta<1, \alpha+\beta>\gamma+\delta$. Пусть $\left\{w_{n}(t)\right\}$ будет набором собственных функиий задачи на собственные значения на интервале $[-\infty, 0]$ для уравнения $(25)$ с набором параметров $\alpha^{\prime \prime}, \beta^{\prime \prime}, \gamma^{\prime \prime}, \delta^{\prime \prime}$, $\varepsilon^{\prime \prime}, q^{\prime \prime}:$

$$
\begin{gathered}
\alpha^{\prime \prime}=2-\alpha, \quad \beta^{\prime \prime}=2-\gamma, \quad \gamma=2-\beta, \\
\delta^{\prime \prime}=\varepsilon+1-\alpha, \quad \varepsilon^{\prime \prime}=\delta+1-\alpha, \quad q^{\prime \prime}=q-a \delta+(2-\gamma)(a+1)-\varepsilon
\end{gathered}
$$

$(w(t)$ ограничена в точках $-\infty$ u 0$)$. Тогда соотношение

$$
y_{n}(z)=S^{(0)} w_{n}(t)=\int_{-\infty}^{0}\left(1-\frac{z s}{a}\right)^{-\alpha} w_{n}(s) d s
$$

порождает набор собственных функций $y_{n}(z)$ для (6), соответствующих задаче на собственные значения на интервале $[1, a]\left(y_{n}(z)\right.$ ограничена в точках 1 и а).

Хотя (33) и (34), в сушности, совпадают, они порождают две лестницы интегральных соотношений для конфлюентных уравнений.

Рассматривая переход $L^{(0)} \mapsto L^{(1)}$, приводяший к процессу конфлюенции, мы получаем интегральное соотношение для конфлюентных функций класса Гойна.

Tеорема 3. Предположим, что $p>0, \delta>\alpha, \gamma>1, \alpha<1$. Пусть $\left\{v_{n}(t)\right\}$ есть набор собственных функиий для задачи на собственные значения на интервале $[0,1]$ для КУГ (7) с параметрами $\alpha^{\prime}=2-\alpha, \quad \gamma^{\prime}=\gamma+1-\alpha, \quad \delta^{\prime}=\delta+1-\alpha$, 
$p^{\prime}=p, q^{\prime}=q-(\alpha-1)(p-\delta-\gamma+\alpha) \quad\left(v_{n}(t)\right.$ ограничена в точках 0 u 1$)$. Тогда соотношение

$$
y_{n}(z)=K^{(1)} v_{n}=\int_{0}^{1}(z-t)^{-\alpha} v_{n}(t) d t
$$

порождает набор собственных функиий $y_{n}(z)$, соответствующих задаче на собственнье значения на интервале $[-\infty, 0]$ для КУГ $(7)\left(y_{n}(z)\right.$ ограничена в точках 0 $u-\infty)$.

ДокАЗАТЕЛЬСТво аналогично доказательству теоремы 1. Обращаясь к БКУГ, можно сформулировать следуюшее предложение.

Теорема 4. Предположим, что $\gamma>1, \quad \alpha<1$. Пусть $\left\{v_{n}(t)\right\}$ есть набор собственных функций задачи на собственные значения на интервале $[0,-\infty]$ для БКУГ (11) с параметрами $\alpha^{\prime}=2-\alpha, \mu^{\prime}=\mu, \gamma^{\prime}=\gamma+1-\alpha, \quad q^{\prime}=q+\mu(\alpha-1)$ $\left(v_{n}(t)\right.$ ограничена в точках $\left.0 u-\infty\right)$. Тогда соотношение

$$
y_{n}(z)=K^{(5)} v_{n}=\int_{0}^{\infty}(z-t)^{-\alpha} v_{n}(t) d t
$$

порождает набор собственнъх функиий $y_{n}(z)$ задачи на собственнье значения на интервале $[-\infty, 0]$ для БКУГ (11) ( $y_{n}(z)$ ограничена в точках $\left.0 u-\infty\right)$.

Отметим, что, как следует из теорем $1-4$, если $\alpha=-n$ и $n$ целое, то собственные функции $y_{n}(z)$ - полиномы.

Обратимся теперь ко второй лестнице интегральных соотношений для конфлюентных функций класса Гойна, порождаемых (34). Первым возникает интегральное соотношение для КУГ, альтернативное (35). Оно может быть получено прямым предельным переходом (15), когда ядро уравнения (34) преобразуется в экспоненту.

Теорема 5. Предположим, что $\delta>1, \beta<1, p<0$. Пусть $\left\{w_{n}(t)\right\}$ есть набор собственных функций задачи на собственные значения на интервале $[-\infty, 0]$ для КУГ (7) с параметрами $\alpha^{\prime}=2-\gamma, \gamma^{\prime}=2-\alpha, \quad \delta^{\prime}=\alpha+2-\gamma-\delta, \quad p^{\prime}=-p$, $q^{\prime}=q+\delta+\gamma-2-p\left(w_{n}(t)\right.$ ограничена в точках $\left.0 u-\infty\right)$. Тогда соотночение

$$
y_{n}(z)=S^{(1)} w_{n}=\int_{-\infty}^{0} \exp (-p z t) w_{n}(t) d t
$$

порождает набор собственных функиий $y_{n}(z)$ двухточечной задачи на собственные значения на интервале $[0, \infty]$ для КУГ $(7),\left(y_{n}(z)\right.$ ограничена в точках $\left.0 u \infty\right)$.

Далее следует интегральное соотношение для БКУГ.

ТЕОрема 6. Предположимм, что $\gamma<1, \quad \beta>1$. Пусть $\left\{w_{n}(t)\right\}$ есть набор собственных функций задачи на собственные значения на интервале $[0, \infty]$ для БKУГ

$$
\left(t D_{t}^{2}+\left(t^{2}+\mu^{\prime} t+\gamma^{\prime}\right) D_{t}+\alpha^{\prime} t-q^{\prime}\right) w(t)=0
$$


ИНТЕГРАЛЬНЫЕ СООТНОШЕНИЯ ДЛЯ СПЕЦИАЛЬНЫХ ФУНКЦИЙ КЛАССА ГОЙНА 395

где $\alpha^{\prime}=2-\gamma, \gamma^{\prime}=2-\alpha, \mu^{\prime}=-\mu, q^{\prime}=q+\mu\left(w_{n}(t)\right.$ ограничена в точках $\left.0 u \infty\right)$. Тогда соотночение

$$
y_{n}(z)=S^{(5)} w_{n}=\int_{0}^{\infty} \exp (-z t) w_{n}(t) d t
$$

порождает набор собственных функиий $y_{n}(z)$ двухточечной задачи на собственные значения на интервале $[0, \infty]$ для уравнения $(11)\left(y_{n}(z)\right.$ ограничена в точках $0 \quad u \infty)$.

Среди соотношений, соответствуюших ДКУГ, мы можем выделить соотношения между решениями редуцированного ДКУГ и соотношения между решениями ДКУГ и решениями редуцированного КУГ.

Во втором случае справедлива

ТЕОрема 7. Предположим, что $\alpha<1, \quad \nu>0$. Пусть $\left\{w_{n}(t)\right\}$ есть набор собственных функиий задачи на собственные значения на интервале $[-\infty, 0]$ для редуцированного КУГ (8) с параметрами $\gamma^{\prime}=2-\alpha, \quad \delta^{\prime}=\alpha+2-\tau, k^{\prime 2}=-\nu$, $q^{\prime}=q+\tau-2(w(t)$ ограничена в точках $0 u-\infty)$. Тогда соотношение

$$
y_{n}(z)=S^{(1)} w_{n}=\int_{-\infty}^{0} \exp (-z t) w_{n}(t) d t
$$

порождает набор собственных функиий $y_{n}(z)$ задачи на собственные значения на интервале $[0, \infty]$ для ДКУГ (9) (yn $(z)$ ограничена в точках $0 u \infty)$.

Теорема 8. Предположим, что $\gamma>1, \delta>1, k^{2}>0$. Пусть $\left\{w_{n}(t)\right\}$ есть набор собственных функций задачи на собственные значения на интервале $[-\infty, 0]$ для ДКУГ (9) с параметрами $\alpha^{\prime}=2-\gamma, \tau^{\prime}=4-\gamma-\delta, \nu^{\prime}=-k^{2}, \quad q^{\prime}=q+\delta+\gamma-2$ $\left(w_{n}(t)\right.$ ограничена в точках $\left.0 u-\infty\right)$. Тогда соотношение

$$
y_{n}(z)=\int_{-\infty}^{0} \exp (-z t) w_{n}(t) d t
$$

порождает набор собственных функиий $y_{n}(z)$ задачи на собственные значения на интервале $[0,1]$ для редуцированного КУГ (8) ( $y_{n}(z)$ ограничена в точках 0 u 1$)$.

ТЕОРема 9. Предположим, что $\omega>0$. Пусть $\left\{w_{n}(t)\right\}$ есть набор собственных функций задачи на собственные значения на интервале $[0, \infty)$ для редуцированного ДКУГ (10) с параметрами $\rho^{\prime}=4-\rho, \omega^{\prime}=\omega, q^{\prime}=\rho-2-q\left(w_{n}(t)\right.$ ограничена в точках 0 и $\infty)$. Тогда соотношение

$$
y_{n}(z)=\int_{0}^{\infty} \exp (-z t / \omega) w_{n}(t) d t
$$

порождает набор собственных функиий $y_{n}(z)$ задачи на собственные значения на интервале $[0, \infty)$ для редучированного ДКУГ (10) $\left(y_{n}(z)\right.$ ограничена в точках 0 $u \infty)$.

В приведенный выше список интегральных соотношений не вошли соотношения, возникаюшие при специальных значениях параметров рассмотренных уравнений. Помимо 
соотношений между собственными функциями тем же способом можно искать соотношения между другими специально выбранными решениями, однако число возможностей здесь слишком велико для журнальной статьи.

Статья написана благодаря финансовой поддержке фонда Copoca, грант R5H000.

Авторы благодарны А.Зеегеру, Д.Шмидту, В.Лаю и В.С. Буслаеву за полезные обсуждения вопросов, затронутых в статье.

\section{Список литературы}

[1] A.Decarreau, M.C.Dumont-Lepage, P.Maroni, A.Robert, A.Ronveaux // Ann. Soc. Sc. de Brux. 1978. V. 92. P. 53-78; A.Decarreau, P.Maroni, A.Robert // Ann. Soc. Sc. de Brux. 1978. V. 92. P. $151-189$.

[2] A.Ronveaux (Ed. ) Heun's Differential Equations: Oxford Univ. Press, 1995.

[3] A.Ya.Kazakov, S.Yu.Slavyanov // Methods and Applications of Analysis (to appear).

[4] S.Yu.Slavyanov, W.Lay, A.Seeger // Heun's Differential Equation: Oxford Univ. Press, 1955.

[5] С.Ю.Славянов, А.Зеегер, В.Лай // ТМФ. 1995. Т. 104. №2. С. 233-247.

Санкт-Петербургский государственный университет

Поступила в редакцию 19.VI.1995 г.

\section{A. Ya. Kazakov, S. Yu. Slavyanov \\ INTEGRAL RELATIONS FOR SPECIAL FUNCTIONS OF HYPERGEOMETRIC AND HEUN CLASS}

Ordinary differential equations with polynomial coefficients originate different kinds of integral relations for its solutions: integral representations in terms of simpler functions, integral equations etc. In this paper, a new kind of integral relations for functions of the Heun class are presented. These relations are coupling in ivolution eigensolutions, which are characterized by different behaviour at singularities and often also by different intervals of consideration and equations themselves. The studied relations are arranged in two staircases where each succeeding equation may be obtained with the help of the confluence process. 